\title{
Tumor suppressor candidate 3 as a novel predictor for lymph node metastasis in lung cancer patients
}

\author{
XINSHUANG YU ${ }^{1}$, KAIXIAN ZHANG ${ }^{2}$, FENGJUN LIU ${ }^{1}$, JIANDONG ZHANG ${ }^{1}$, CHUNJUAN ZHAI ${ }^{3}$, \\ LILI CAO ${ }^{4}$, XINGYE SONG ${ }^{5}$, YAO WANG ${ }^{4}$, BAOSHENG LI $^{6}, \mathrm{HONGJUN} \mathrm{SUN}^{4}$ and JUAN DU JUA $^{1,4}$ \\ ${ }^{1}$ Department of Radiation Oncology, Shandong Provincial Qianfoshan Hospital, Shandong University, Jinan, \\ Shandong 250014; ${ }^{2}$ Department of Radiation Oncology, Tengzhou Central People Hospital, Tengzhou, Shandong 277500; \\ ${ }^{3}$ Department of Cardiology, Shandong Provincial Hospital Affiliated to Shandong University, Jinan, Shandong 250021; \\ ${ }^{4}$ Medical Research Center, Shandong Provincial Qianfoshan Hospital, Shandong University, Jinan, Shandong 250014; \\ ${ }^{5}$ Health Center of the Third Sanatorium, Jinan Military Area Command, Jinan, Shandong 250002; \\ ${ }^{6}$ Sixth Department of Radiation Oncology, Shandong Cancer Hospital, Jinan, Shandong 250117, P.R. China
}

Received May 26, 2015; Accepted October 5, 2016

DOI: $10.3892 / \mathrm{ol} .2016 .5333$

\begin{abstract}
Tumor suppressor candidate 3 (TUSC3) was recently identified as a potential tumor suppressor gene in several cancer types. However, no data are currently available regarding the expression of TUSC 3 in lung cancer. The present study investigated the expression of TUSC3 in patients with lung cancer and determined its association with the clinicopathological parameters of the disease. Cytoplasmic TUSC3 expression was evaluated by immunohistochemistry on tissue microarray slides, which included 35 small cell lung cancer (SCLC) specimens, 80 squamous cell lung cancer specimens (SCC), 80 adenocarcinoma lung cancer (ADC) specimens and 37 normal lung tissue specimens. Analysis showed significantly reduced TUSC3 expression in the SCLC patients, but not in the ADC and SCC patients, as compared with the normal controls. Additionally, TUSC3 expression in the patients with a degree of differentiation of 1-2 (well-moderately differentiated) was significantly higher than that in patients with a differentiation degree of 3-4 (poorly differentiated-undifferentiated). Further analysis showed that TUSC3 expression levels were
\end{abstract}

Correspondence to: Dr Juan Du or Dr Hongjun Sun, Medical Research Center, Shandong Provincial Qianfoshan Hospital, Shandong University, 16766 Jingshi Road, Jinan, Shandong 250014, P.R. China

E-mail: sunnydujuan@aliyun.com

E-mail: sunhongjun@sdhospital.com.cn

Abbreviations: TUSC3, tumor suppressor candidate 3; IHC, immunohistochemistry; SCLC, small cell lung cancer; SCC, squamous lung cancer; ADC, adenocarcinoma lung cancer; NSCLC, non-SCLC

*Contributed equally

Key words: tumor suppressor candidate 3, lymph node metastasis, degree of differentiation, immunohistochemistry negatively correlated with the degree of differentiation in the ADC and SCC patients. Notably, a marked decrease in TUSC3 expression was identified in the patients who were lymph node metastasis-positive $\left(\mathrm{LNM}^{+}\right)$compared with patients who were

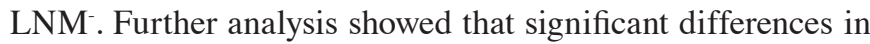
TUSC 3 expression were identified among the different $\mathrm{N}$ stages (LNM status) in the SCLC, ADC and SCC patients. Correlation analysis also identified a negative correlation between TUSC3 expression and LNM in all three pathological types of lung cancer tested. Overall, these results indicated that a reduction in TUSC3 may be associated with a poorly-differentiated grade of lung cancer. Importantly, TUSC3 expression may be a useful predictor of LNM in lung cancer patients. A combined analysis of TUSC3 expression and the clinical variables will aid in predicting the incidence of LNM.

\section{Introduction}

Lung cancer accounts for $70-80 \%$ of cancer-associated mortalities worldwide (1). Lung cancers are classified according to histological type (2) in clinical practice. For therapeutic purposes, there are two broad classes: Small cell lung carcinoma (SCLC) and non-SCLC (3). Adenocarcinoma (ADC) and squamous-cell carcinoma (SCC) are the two major subtypes of NSCLC, and account for 40 and $30 \%$ of lung cancer cases, respectively (2). In SCLC, the malignant cancer cells contain dense neurosecretory granules (vesicles containing neuroendocrine hormones), which render SCLC an endocrine/paraneoplastic-associated tumor, and tumor malignance is attributed to this hormone production (4). Although complete surgical resection can be particularly important in the treatment of lung cancer, only a limited number of patients undergo curative surgery, as lung cancer is most commonly diagnosed at an advanced stage, at which point patients are unable to undergo a surgical procedure (5). Lung cancer is extremely aggressive and often results in a poor prognosis. An improved understanding of lung cancer is urgently required to identify novel drug targets and effective therapeutic strategies for lung cancer patients. 
Tumor suppressor candidate 3 (TUSC3), originally termed $\mathrm{N} 33$, has been known to be responsible for autosomal recessive mental retardation for several years (6-8). Only recently was TUSC3 identified as a potential tumor suppressor gene when it was found deleted in several cancer types, including prostate (9) and liver (10) cancer. However, the manner in which TUSC3 functions as a tumor suppressor remains unclear. TUSC3 is expressed in the majority of non-lymphoid human tissues, including the prostate, lungs, liver and colon tissue (11). The protein is localized in the endoplasmic reticulum and encodes a subunit of the endoplasmic reticulum-bound oligosaccharyl transferase (OST) complex, which is primarily responsible for protein N-linked glycosylation (12). While several studies have focused on the correlation between TUSC 3 expression and tumor development (13-16), no data are currently available regarding the expression of TUSC 3 in lung cancer. In the present study, the expression of TUSC 3 in lung cancer, and the association between TUSC 3 expression and the clinicopathological parameters of the disease were investigated.

\section{Materials and methods}

Tissue samples. Tissue microarray slides were purchased from Xi'an Alena Biotechnology Ltd., Co., (Xi'an, China). The slides included 35 SCLC specimens, 80 SCC specimens, 80 ADC specimens and 37 normal lung tissue specimens. The detailed clinicopathological characteristics of the patients with lung cancer are listed in Table I. All patients were clinically staged [tumor-node-metastasis (TNM) staging] according to the seventh edition of the American Joint Committee on Cancer system for lung cancer (2). The degrees of pathological differentiation were defined as follows: 1, Well-differentiated carcinoma; 2 , moderately-differentiated carcinoma; 3 , poorly-differentiated carcinoma; and 4, undifferentiated carcinoma. The degree of differentiation for the tumors in each of the patients was evaluated by two pathologists. Written informed consent was obtained from all patients, and the study was approved by the Ethical Committe of Shandong Provincial Qianfoshan Hospital (Shandong University, Jinan, China).

Immunohistochemistry (IHC) assay. IHC staining was performed directly on the $5-\mu \mathrm{m}$ tissue slides. Briefly, following incubation for $2 \mathrm{~h}$ at $56^{\circ} \mathrm{C}$, the slides were dewaxed with xylene and rehydrated using a graded alcohol series $(100,90,70$ and $50 \%$ ethanol; $5 \mathrm{~min}$ each). Endogenous peroxidase activity was blocked with $3 \% \mathrm{H}_{2} \mathrm{O}_{2}$ for $15 \mathrm{~min}$. For antigen retrieval, the sections were incubated in sodium citrate buffer $(0.01 \mathrm{M}$, $\mathrm{pH}$ 6.0) for $20 \mathrm{~min}$ in a household microwave oven $(600 \mathrm{~W})$. Next, the slides were incubated with $10 \%$ normal goat serum to block non-specific binding sites. Thereafter, the slides were incubated with the TUSC 3 goat polyclonal antibody (1:100 final dilution; catalog no. sc-98191; Santa Cruz, USA) overnight at $4^{\circ} \mathrm{C}$. Subsequent to washing the slides with phosphate-buffered saline, the bio-labeled secondary antibody, rabbit anti-goat IgG (catalog no. ZDR 5308; ZSGB-Bio, Beijing, China), was applied at a 1:200 dilution for $40 \mathrm{~min}$ at $37^{\circ} \mathrm{C}$. The sections were then stained with diaminobenzidine. Finally, the sections were counterstained with hematoxylin and eosin, dehydrated with graded alcohol and mounted using neutral gum. Stained cell scoring using a digital pathology system was performed by Aperio ImageScope (Aperio Technologies, Inc., Vista, CA, USA).

Immunoreactivity was observed in the cytoplasm of the cells and the scoring was based on cytoplasmic staining. Immunoreactivity for TUSC 3 expression was independently evaluated by three pathologists from the Shandong Provincial Qianfoshan Hospital, and categorized according to the immunoreactive score (IRS) as follows: IRS = staining intensity (SI) x percentage of positively stained cells (PP). SI was determined as 0 (negative), 1 (weak), 2 (moderate) or 3 (strong). PP was scored as 0 (negative), 1 ( $<25 \%$ of the cells), 2 (25-50\% of the cells), 3 (50-75\% of the cells) or 4 ( $>75 \%$ of the cells). A final score was then calculated by multiplying these two scores. Additionally, all the specimens were divided into two groups, showing negative or positive expression, using an IRS of 6 as the cut-off value.

Statistical analysis. The SPSS 13.0 software (SPSS, Inc., Chicago, IL, USA) was used for the statistical analyses. Levels of TUSC 3 expression were compared using a rank sum test. Comparisons of the positive rates between two groups were performed using Fisher's exact test, and Spearman's correlation method was used to evaluate the association of scores. The significance of the correlation between clinical pathological parameters (age, gender, grading, T-stage, $\mathrm{N}$-stage and differentiation degree) and IRS of TUSC3 was determined using Fisher's exact test. All reported P-values were two-sided, and $\mathrm{P}<0.05$ was considered to indicate a statistically significant difference.

\section{Results}

Patient characteristics. The basic characteristics of the patients are shown in Table I. The median age of the healthy controls was 53 years (range, 26-76 years) and that of lung cancer patients was 56 years (range, 16-76 years). No significant difference was observed in gender or age between the normal controls and patients. Regarding the TNM staging, a significant decrease in TUSC3 expression could be observed in the patients with stage III+IV disease compared with those with stage I+II disease ( $\mathrm{P}=0.027$; Table I). When lymph node metastasis (LNM) was considered, analysis revealed a marked decrease in TUSC 3 expression in the patients who were $\mathrm{LNM}^{+}$ compared with patients who were $\mathrm{LNM}^{-}(\mathrm{P}=0.011$, Table I). Additionally, the positive rate of TUSC 3 expression in the patients with a differentiation degree of 1-2 was significantly higher than that in the patients with a differentiation degree of 3-4 ( $\mathrm{P}<0.001$; Table I). The representative IHC assay is shown in Fig. 1.

General expression levels in normal controls and cancer patients. The positive rates of TUSC 3 expression in the normal controls and lung cancer patients were 59.5\% (22/37) and $45.6 \%$ (89/195), respectively (Table II). There was no significant difference between the normal controls and lung cancer patients with regard to the TUSC 3 expression rate $\left(\chi^{2}=0.238\right.$, $\mathrm{P}=0.123$; Table II). When the patients were grouped according to clinical classification, i.e., the SCLC, SCC, ADC and normal control group, differences in TUSC 3 expression were identified among multiple groups (normal vs. SCLC, $\mathrm{P}=0.0005$; normal vs. $\mathrm{ADC}, \mathrm{P}=0.3250$; normal vs. $\mathrm{SCC}, \mathrm{P}=0.0546$; 
Table I. Basic characteristics of the patients.

\begin{tabular}{|c|c|c|c|c|c|c|}
\hline Characteristic & Number $(\%)$ & Positive, $\mathrm{n}$ & Negative, $\mathrm{n}$ & Positive rate, $\%$ & $\chi^{2}$ & P-value ${ }^{a}$ \\
\hline Age, years ${ }^{b}$ & & & & & 0.691 & 0.406 \\
\hline$<56$ & $90(46.2)$ & 39 & 61 & 43.3 & & \\
\hline$\geq 56$ & $105(53.8)$ & 50 & 65 & 47.6 & & \\
\hline Gender & & & & & 0.239 & 0.625 \\
\hline Male & $144(73.8)$ & 63 & 93 & 43.8 & & \\
\hline Female & $51(26.2)$ & 26 & 33 & 51.0 & & \\
\hline Histological type & & & & & 11.304 & 0.001 \\
\hline SCLC & 35 (17.9) & 7 & 28 & 20.0 & & \\
\hline NSCLC & $160(82.1)$ & 82 & 78 & 51.3 & & \\
\hline TNM staging $^{\mathrm{c}}$ & & & & & 4.903 & 0.027 \\
\hline $\mathrm{I}+\mathrm{II}$ & $76(39.0)$ & 39 & 37 & 51.3 & & \\
\hline $\mathrm{III}+\mathrm{IV}$ & $119(61.0)$ & 42 & 77 & 35.3 & & \\
\hline LNM & & & & & 6.459 & 0.011 \\
\hline Negative & $65(33.3)$ & 38 & 27 & 58.5 & & \\
\hline Positive & $130(66.7)$ & 51 & 79 & 39.2 & & \\
\hline Degree of differentiation ${ }^{\mathrm{d}}$ & & & & & 21.817 & 0.000 \\
\hline $1-2$ & $144(73.8)$ & 80 & 64 & 55.6 & & \\
\hline $3-4$ & $51(26.2)$ & 9 & 42 & 17.6 & & \\
\hline
\end{tabular}

aPositive rates of tumor suppressor candidate 3 expression were compared by Fisher's exact test. ${ }^{b}$ Median age, 56 years (range, 16-76 years). 'TNM staging is defined according to the seventh edition of the TNM classification for malignant tumors. ${ }^{\mathrm{d}}$ Degree of differentiation was evaluated by two pathologists from Shandong Provincial Qianfoshan Hospital (Shandong University, Jinan, China). The pathological degree of differentiation was defined as follows: 1, Well-differentiated carcinoma; 2, moderately-differentiated carcinoma; 3, poorly-differentiated carcinoma; and 4, undifferentiated carcinoma. SCLC, small cell lung cancer; LNM, lymph node metastasis; NSCLC, non-SCLC (including adenocarcinoma and squamous carcinoma); TNM, tumor-node-metastasis.

$\mathbf{A}$

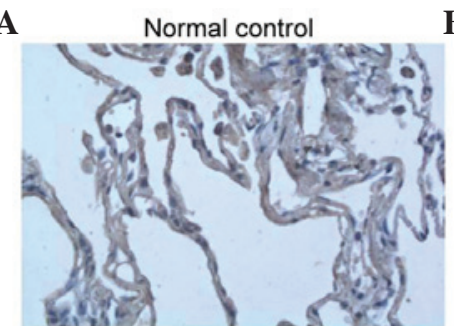

B SCLC

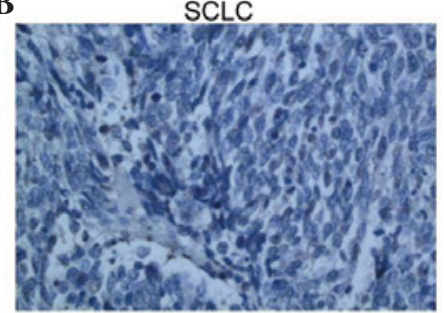

C Poorly-differentiated ADC
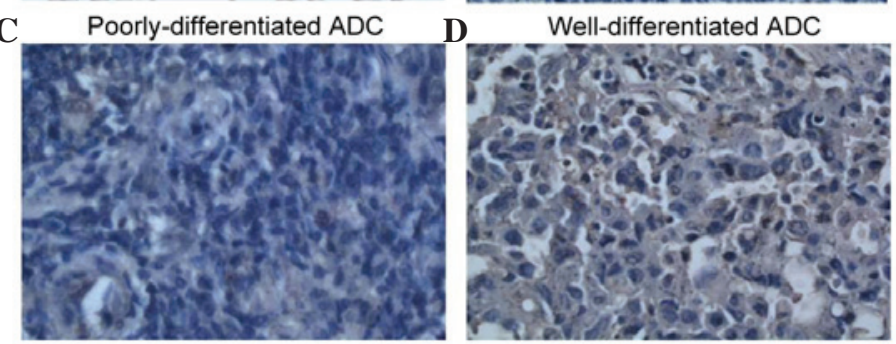

E

Poorly-differentiated SCC

Well-differentiated SCC
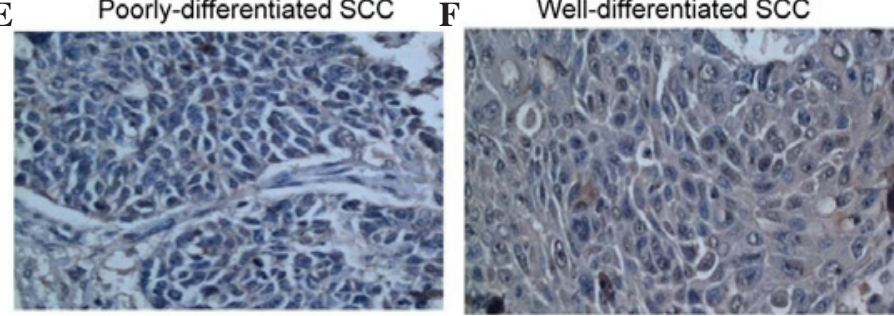

Figure 1. Immunohistochemical staining of tumor suppressor candidate 3 in human lung cancers (magnification, x40). (A) Normal control; (B) SCLC; (C) poorly-differentiated ADC; (D) well-differentiated ADC; (E) poorly-differentiated SCC; (F) well-differentiated SCC. SCLC, small cell lung carcinoma; ADC, adenocarcinoma; SCC, squamous cell carcinoma. 
Table II. Comparison of TUSC3 expression between normal controls and lung cancer patients.

\begin{tabular}{|c|c|c|c|c|c|c|}
\hline \multirow[b]{2}{*}{ Patient type } & \multirow[b]{2}{*}{ Number } & \multicolumn{2}{|c|}{ TUSC 3 expression, $\mathrm{n}$} & \multirow{2}{*}{$\begin{array}{l}\text { Positive } \\
\text { rate, } \%\end{array}$} & \multirow[b]{2}{*}{$\chi^{2}$} & \multirow[b]{2}{*}{ P-value ${ }^{a}$} \\
\hline & & + & - & & & \\
\hline Normal control & 37 & 22 & 15 & 59.5 & 0.238 & 0.123 \\
\hline Lung cancer ${ }^{b}$ & 195 & 89 & 106 & 45.6 & & \\
\hline
\end{tabular}

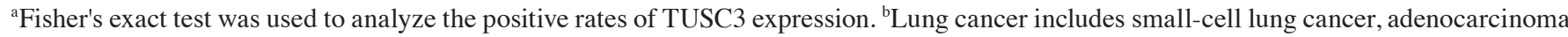
and squamous carcinoma. $\mathrm{P}<0.05$ was considered to indicate statistical significance. TUSC 3 , tumor suppressor candidate 3.

Table III. Comparison of TUSC 3 expression between normal controls and SCLC patients.

\begin{tabular}{lcccccc}
\hline & & \multicolumn{2}{c}{ TUSC3 expression, $n$} & & Positive \\
Patient type & Number & + & - & & $\chi^{2}$ & P-value $^{\mathrm{a}}$ \\
\cline { 3 - 6 } & 37 & 22 & 15 & 59.5 & 11.642 & 0.001 \\
Normal & 35 & 7 & 28 & 20.0 & & \\
\hline
\end{tabular}

${ }^{a}$ Fisher's exact test was used to analyze the positive rates of TUSC3 expression. $\mathrm{P}<0.05$ was considered to indicate statistical significance. TUSC3, tumor suppressor candidate 3; SCLC, small cell lung cancer.

Table IV. Comparison of TUSC3 expression between normal controls and ADC patients.

\begin{tabular}{|c|c|c|c|c|c|c|}
\hline \multirow[b]{2}{*}{ Patient type } & \multirow[b]{2}{*}{ Number } & \multicolumn{2}{|c|}{ TUSC 3 expression, $n$} & \multirow{2}{*}{$\begin{array}{c}\text { Positive } \\
\text { rate, } \%\end{array}$} & \multirow[b]{2}{*}{$\chi^{2}$} & \multirow[b]{2}{*}{ P-value ${ }^{a}$} \\
\hline & & + & - & & & \\
\hline Normal & 37 & 22 & 15 & 59.5 & 2.499 & 0.114 \\
\hline $\mathrm{ADC}$ & 80 & 47 & 33 & 58.8 & & \\
\hline
\end{tabular}

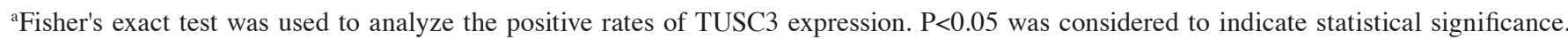
TUSC3, tumor suppressor candidate 3; ADC, adenocarcinoma.

SCLC vs. ADC, P=0.0012; SCLC vs. SCC, P=0.0636; SCC vs. $\mathrm{ADC}, \mathrm{P}=0.6490$ ) (rank sum; Fig.2). Moreover, the positive rate of TUSC 3 expression in the SCLC patients was significantly lower than that in the normal controls $\left(\chi^{2}=11.642, \mathrm{P}=0.001\right.$; Table III). However, the positive rate of TUSC3 expression showed no significant difference between the normal controls and the ADC patients $\left(\chi^{2}=2.499, \mathrm{P}=0.114\right.$; Table IV $)$, or between the controls and the SCC patients $\left(\chi^{2}=0.255, \mathrm{P}=0.614\right.$; Table V). Similarly, no significant difference in the positive rate of TUSC 3 was observed between the ADC and SCC groups $\left(\chi^{2}=3.602, \mathrm{P}=0.058\right.$; Table VI).

Association between TUSC3 expression and the TNM staging of lung cancer patients. As shown in Fig. 3, there were significant differences in TUSC 3 expression among the patients with different TNM staging (stages I-IV) in the SCLC $(\mathrm{P}=0.0006$; Fig. $3 \mathrm{~A})$ and ADC $(\mathrm{P}<0.0001$; Fig. 3D) patients, but not in the $\mathrm{SCC}$ patients $(\mathrm{P}=0.216$; Fig. $3 \mathrm{H})$. Additionally, significant differences in TUSC3 expression were identified among the patients with different $\mathrm{N}$ stages in the SCLC ( $\mathrm{P}=0.0012$; Fig. 3C), ADC ( $\mathrm{P}<0.0001$; Fig. 3F) and SCC

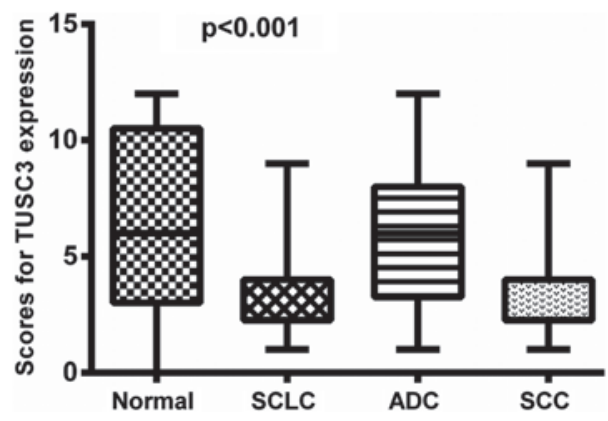

Figure 2. TUSC 3 expression was compared among different patient groups. The rank sum test was used to analyze the differences among multiple groups. Normal, normal controls; SCLC, small cell lung carcinoma; ADC, adenocarcinoma; SCC, squamous cell carcinoma; TUSC3, tumor suppressor candidate 3 .

patients $(\mathrm{P}<0.0001$; Fig. 3J $)$. However, no such differences were found among the patients with different $\mathrm{T}$ stages in the SCLC ( $\mathrm{P}=0.208$; Fig. 3B), ADC ( $\mathrm{P}=0.208$; Fig. 3E) and SCC patients $(\mathrm{P}=0.641$; Fig. 3I). 
Table V. Comparison of TUSC3 expression between normal controls and SCC patients.

\begin{tabular}{|c|c|c|c|c|c|c|}
\hline \multirow[b]{2}{*}{ Patient type } & \multirow[b]{2}{*}{ Number } & \multicolumn{2}{|c|}{ TUSC 3 expression, $\mathrm{n}$} & \multirow{2}{*}{$\begin{array}{c}\text { Positive } \\
\text { rate, } \%\end{array}$} & \multirow[b]{2}{*}{$\chi^{2}$} & \multirow[b]{2}{*}{ P-value ${ }^{a}$} \\
\hline & & + & - & & & \\
\hline Normal & 37 & 22 & 15 & 59.5 & 0.255 & 0.614 \\
\hline SCC & 80 & 35 & 45 & 43.8 & & \\
\hline
\end{tabular}

${ }^{\text {a}}$ Fisher's exact test was used to analyze the positive rates of TUSC3 expression. $\mathrm{P}<0.05$ was considered to indicate statistical significance. TUSC3, tumor suppressor candidate 3; SCC, squamous carcinoma.

Table VI. Comparison of TUSC3 expression between ADC and SCC patients.

\begin{tabular}{|c|c|c|c|c|c|c|}
\hline \multirow[b]{2}{*}{ Patient type } & \multirow[b]{2}{*}{ Number } & \multicolumn{2}{|c|}{ TUSC 3 expression, $\mathrm{n}$} & \multirow{2}{*}{$\begin{array}{l}\text { Positive } \\
\text { rate, \% }\end{array}$} & \multirow[b]{2}{*}{$\chi^{2}$} & \multirow[b]{2}{*}{ P-value ${ }^{a}$} \\
\hline & & + & - & & & \\
\hline $\mathrm{ADC}$ & 80 & 47 & 33 & 58.8 & 3.602 & 0.058 \\
\hline $\mathrm{SCC}$ & 80 & 35 & 45 & 43.8 & & \\
\hline
\end{tabular}

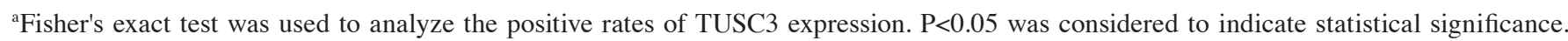
TUSC3, tumor suppressor candidate 3; ADC, adenocarcinoma; SCC, squamous carcinoma.
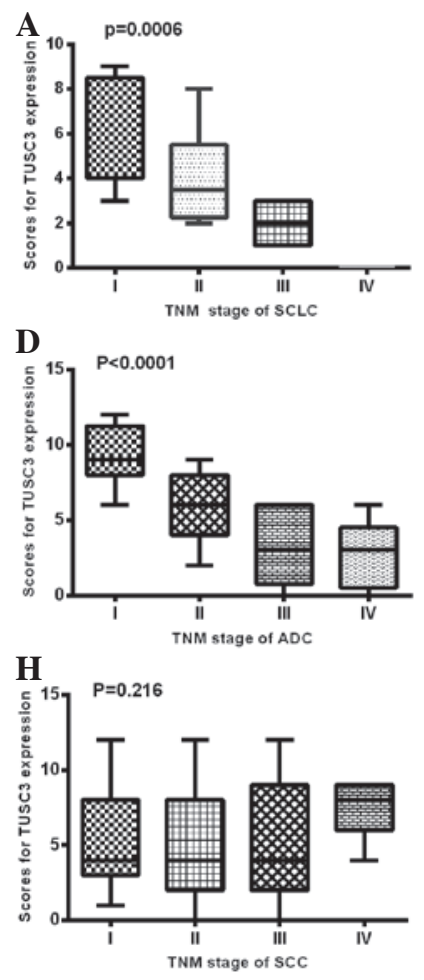
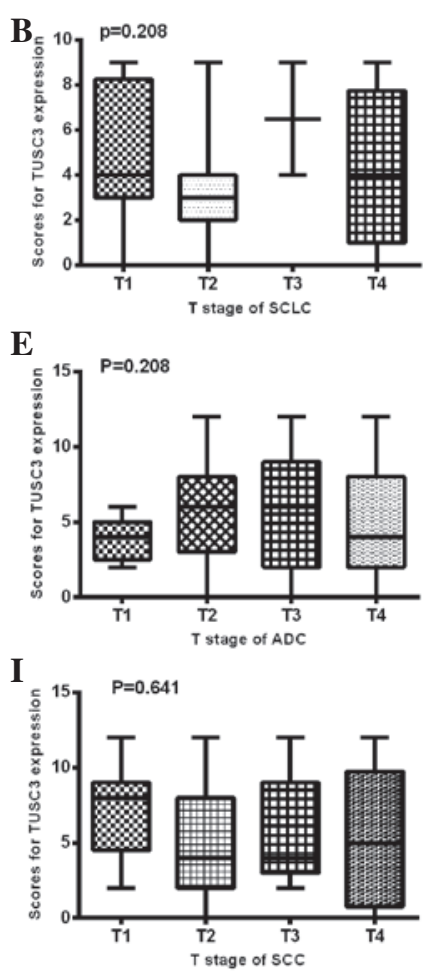

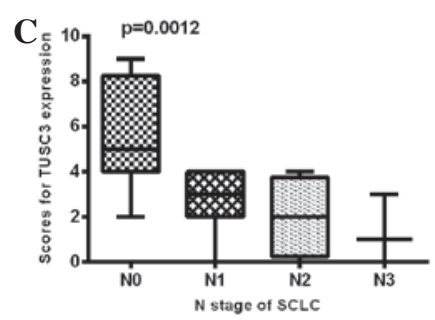

$\mathbf{F}$
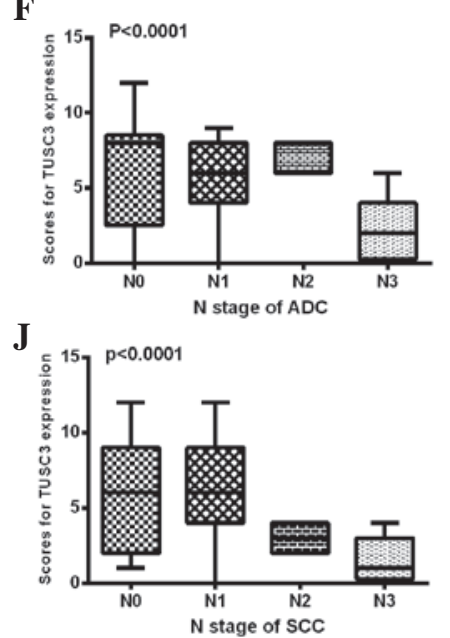

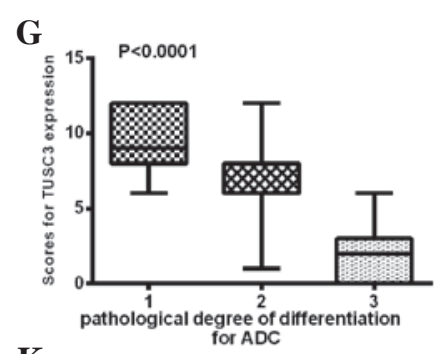

$\mathbf{K}$

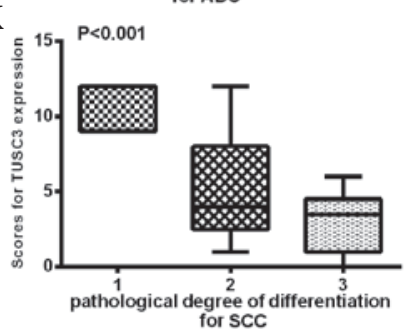

Figure 3. TUSC3 expression was compared among different clinical TNM stages and pathological degrees of differentiation in three types of lung cancer patients. In (A-C) SCLC, (D-G) ADC and (H-K) SCC patients, TUSC3 expression was compared among different (A, D and E) clinical TNM stages, $(\mathrm{B}, \mathrm{E}$ and $\mathrm{I}) \mathrm{T}$ stages, $(\mathrm{C}, \mathrm{F}$ and $\mathrm{J}) \mathrm{N}$ stages, and $(\mathrm{G}$ and $\mathrm{K})$ pathological degrees of differentiation. The rank sum test was used to analyze the differences between groups. SCLC, small cell lung cancer; ADC, adenocarcinoma; SCC, squamous cell carcinoma; TUSC3, tumor suppressor candidate 3.

Further analysis showed that TUSC3 expression was negatively correlated with clinical TNM staging in the SCLC $(\mathrm{P}<0.0001 ; \mathrm{rs}=0.463 ;$ Fig. $4 \mathrm{~A})$ and ADC $(\mathrm{P}<0.0001 ; \mathrm{rs}=0.5245$; Fig. 4D) patients, but not in the SCC patients $(\mathrm{P}=0.173$; rs=0.024;
Fig. 4H). Additionally, TUSC3 expression was negatively correlated with $\mathrm{N}$ stage in the SCLC $(\mathrm{P}<0.0001 ; \mathrm{rs}=0.3835 ;$ Fig. 4C), $\operatorname{ADC}(\mathrm{P}=0.0002$; rs=0.167; Fig. $4 \mathrm{~F})$ and $\mathrm{SCC}(\mathrm{P}=0.002$; $\mathrm{rs}=0.119$; Fig. $4 \mathrm{~J})$ patients. However, there were no correlations 

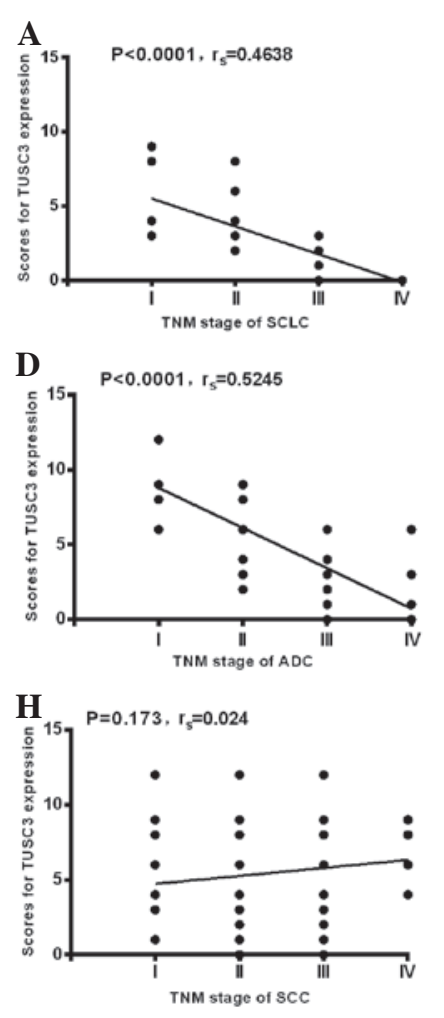

B

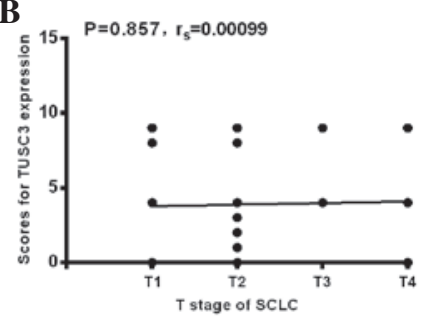

E

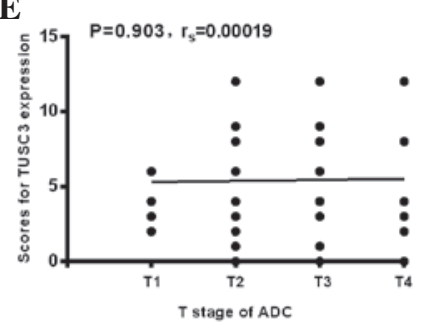

I

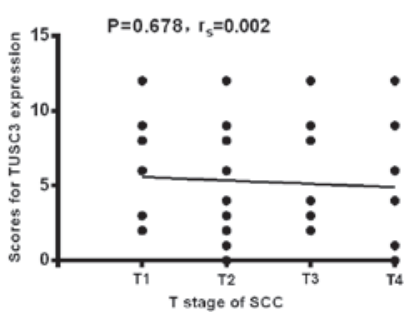

C

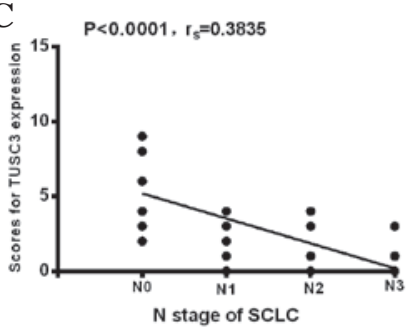

F

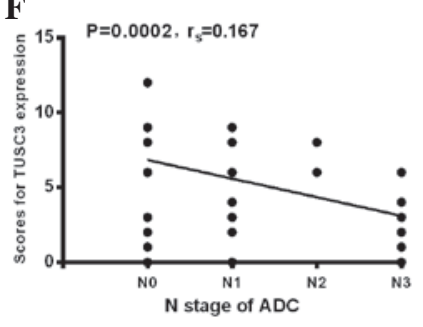

J

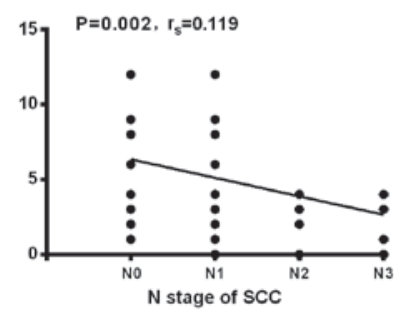

G

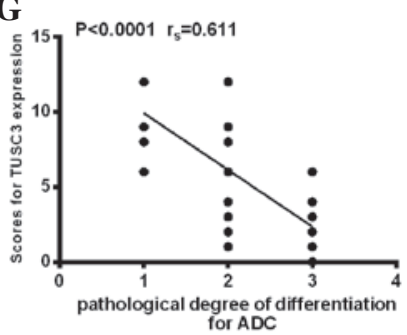

$\mathbf{K}$

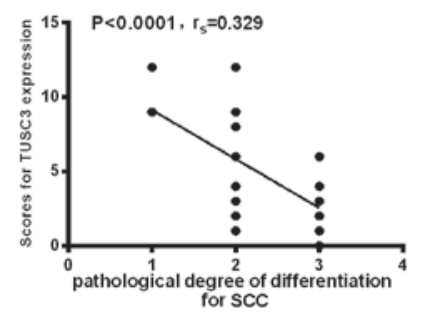

Figure 4. Correlation between TUSC3 expression and different clinical TNM stages or pathological degrees of differentiation in three types of lung cancer patients. In (A-C) SCLC, (D-G) ADC and (H-K) SCC patients, correlations between TUSC3 expression and (A, D and H) clinical TNM, (B, E and I) T stage, (C, F and J) N stage and ( $\mathrm{G}$ and $\mathrm{K}$ ) degree of differentiation were analyzed. Spearman's correlation method was used to evaluate the association of scores. SCLC, small cell lung cancer; ADC, adenocarcinoma; SCC, squamous cell carcinoma; TUSC3, tumor suppressor candidate 3.

between TUSC 3 expression and $\mathrm{T}$ stage in the $\operatorname{SCLC}(\mathrm{P}=0.857$; rs $=0.00099$; Fig. 4B), ADC ( $\mathrm{P}=0.903$; rs=0.00019; Fig. 4E) or $\mathrm{SCC}(\mathrm{P}=0.678 ;$ rs $=0.002$; Fig. $4 \mathrm{I})$ patients.

Association between TUSC3 expression and the pathological differentiation of lung cancer patients. The analysis revealed significant differences between TUSC3 expression and pathological differentiation in ADC $(\mathrm{P}<0.0001$; Fig. 3G) and SCC $(\mathrm{P}<0.001$; Fig. 3K) patients. Moreover, correlation analysis showed that there were negative correlations between TUSC 3 expression and pathological differentiation in the ADC $(\mathrm{P}<0.0001 ; \mathrm{rs}=0.611$; Fig. 4G) and SCC $(\mathrm{P}<0.0001 ; \mathrm{rs}=0.3289$; Fig. $4 \mathrm{~K}$ ) patients. As all SCLC patients presented with a pathological degree of differentiation of 4 (undifferentiated), the correlation between TUSC 3 expression and pathological degree of differentiation was not analyzed in the SCLC patients.

\section{Discussion}

Lung cancer presents with a high mortality rate due mainly to its late diagnosis $(17,18)$. In total, $>75 \%$ of lung cancer cases are diagnosed once the disease has become locally advanced or metastatic, resulting in a current 5-year survival rate of $<15 \%$ (17). Therefore, there is an urgent requirement for reliable predictors and indicators of diagnosis and prognosis for lung cancer.

TUSC3, a subunit of the human endoplasmic reticulum-bound OST complex, has been recognized as a candidate tumor suppressor gene involved in the tumor development of multiple tumors. Loss of TUSC3 slows glycoprotein folding, and induces proliferation, migration and invasion of cancerous cells during tumor progression $(13,16)$. The clinical significance of TUSC 3 expression levels have been determined in several human tumors. Guervós et al (13) found that TUSC3 plays a role in metastasis in larynx and pharynx squamous cell carcinomas, and that the loss of TUSC 3 is negatively correlated with LNM and survival rate. Pils et al (14) found that TUSC3 loss may facilitate tumor growth. Reconstitution of TUSC3 in vitro decreases proliferation and the binding of cancer cells to the extracellular matrix. Therefore, TUSC3 represents a potential predictive factor for survival. Khalid et al (15) found that TUSC3 is involved in testicular spermatogenesis, and that it acts in the normal development of the prostate and in the suppression of tumors. Horak et al (16) found that TUSC3 expression is frequently lost in prostate cancer cell lines, leading to the increased proliferation, migration and invasion of cancer cells. However, the significance of TUSC3 expressions in lung cancer patients has not yet been reported. To the best of our knowledge, the present study is the first to analyze the association between TUSC 3 expression and the clinicopathological parameters of lung cancer.

The study showed there was no significant differences between normal controls and lung cancer patients in terms of TUSC 3 expression rate $\left(\chi^{2}=0.238, P=0.123\right.$; Table II). The results may be due to the heterogeneity of different types of lung cancer. Therefore, the TUSC 3 expression rate was analyzed in SCLC, ADC and SCC patients, respectively. The analysis showed decreased expression of TUSC 3 in the 
SCLC patients compared with the normal controls $(\mathrm{P}=0.001$; Table III). However, no difference in TUSC3 expression was identified between the normal controls and the ADC patients $\left(\chi^{2}=2.499, \mathrm{P}=0.114\right.$; Table IV), or between the controls and the SCC patients $\left(\chi^{2}=0.255, \mathrm{P}=0.614\right.$; Table $\left.\mathrm{V}\right)$. When ADC and SCC were considered together as NSCLC according to the histological type, TUSC3 expression in the SCLC patients was significantly lower than that in the NSCLC patients $(\mathrm{P}=0.001$; Table I). The aforementioned results indicated that decreased TUSC3 expression may play a more significant role in the tumorigenesis of SCLC than in that of ADC and SCC. However, a larger sample size will be used in an upcoming study of lung cancer patients, with a focus on ADC and SCC patients.

Additionally, the association between TUSC3 expression and the pathological degree of differentiation was analyzed. The TUSC3 expression in the patients with a pathological differentiation degree of 1-2 was significantly higher than that in the patients with a differentiation degree of 3-4 ( $<<0.001$; Table I). Further analysis showed that TUSC3 expression levels were negatively correlated with the pathological degree of differentiation in ADC $(\mathrm{P}<0.001$; rs $=0.611 ;$ Fig. $4 \mathrm{G})$ and $\mathrm{SCC}(\mathrm{P}<0.001$; rs=0.3289; Fig. $4 \mathrm{~K}$ ) patients. The correlation between TUSC3 expression and pathological degree of differentiation could not be analyzed in the SCLC patients, as all SCLC patients presented with an differentiation degree of 4 (undifferentiated). Together, these results indicated that decreased TUSC3 expression levels may be associated with a poorly-differentiated tumor grade.

The correlations between TUSC3 expression and clinical TNM staging were evaluated. Significant differences in TUSC3 expression were identified among patients with different TNM stages (stage I-IV) in the SCLC ( $\mathrm{P}=0.0006$; Fig. 3A) and ADC ( $\mathrm{P}<0.0001$; Fig. 3D) patients, but not in the SCC patients $(\mathrm{P}=0.216$; Fig. $3 \mathrm{H})$. Further correlation analysis also confirmed these results (Fig. 4A, D and H). It is known that TNM staging is an index to reflect tumor progression in clinical practice (2). TUSC3 expression may similarly be a useful predictor of the progression of SCLC and ADC. However, this conclusion cannot be obtained in SCC patients. Our further study will verify the aforementioned results using a larger sample size.

Notably, in the present study, the analysis revealed a marked decrease in TUSC3 expression in patients who were $\mathrm{LNM}^{+}$compared with the expression level in patients who were $\mathrm{LNM}^{-}(\mathrm{P}=0.011$; Table I). Furthermore, significant differences in TUSC3 expression were identified among the different $\mathrm{N}$ stages (LNM status) in SCLC $(\mathrm{P}=0.0012$; Fig. 3C), ADC ( $\mathrm{P}<0.0001$; Fig. 3F) and SCC $(\mathrm{P}<0.0001$; Fig. 3J) patients. Similarly, correlation analysis also identified a negative correlation between TUSC3 expression and LNM in all the three pathological types of lung cancer tested (Fig. 4C, F and J). The results suggested that lower TUSC3 expression may indicate a higher probability of LNM in lung cancer patients. These results have significance in clinical practice. For an individual patient, a combined analysis of TUSC3 expression and the clinical variables will assist in predicting the incidence of LNM.

In conclusion, the present findings provide the first evidence that a loss or reduction of TUSC3 may be associated with a poorly-differentiated grade of lung cancer. Notably, TUSC3 may be a novel predictor of LNM in lung cancer patients.

\section{Acknowledgements}

This study was supported by grants from the Natural Science Foundation of Shandong Province (grant nos. ZR2011HQ010 and ZR2015HM077), the Technology Development Plan of Shandong Province (grant no.2015WSB04012) and the National Natural Science Foundation of China (grant no. 30901712).

\section{References}

1. Herbst RS, Heymach JV and Lippman SM: Lung cancer. N Engl J Med 359: 1367-1380, 2008.

2. Ettinger DS, Akerley W, Borghaei H, Chang AC, Cheney RT, Chirieac LR, D'Amico TA, Demmy TL, Govindan R, Grannis FW Jr, et al: Non-small cell lung cancer. Version 2.2013. J Natl Compr Canc Netw 11: 645-653; quiz 653, 2013.

3. Kumar V, Abbas AK and Aster JC: Robbins basic pathology. 9th edition. Elsevier, Saunders, PA, pp505-505, 2013.

4. Rosti G, Bevilacqua G, Bidoli P, Portalone L, Santo A and Genestreti G: Small cell lung cancer. Ann Oncol 17 (Suppl 2): ii5-ii10, 2006.

5. Chute JP, Chen T, Feigal E, Simon R and Johnson BE: Twenty years of phase III trails for patients with extensive-stage small-cell lung cancer: Perceptible progress. J Clin Oncol 17: 1794-1801, 1999.

6. Loddo S, Parisi V, Doccini V, Filippi T, Bernardini L, Brovedani P, Ricci F, Novelli A and Battaglia A: Homozygous deletion in TUSC 3 causing syndromic intellectual disability: A new patient. Am J Med Genet A 161A: 2084-2087, 2013.

7. Garshasbi M, Kahrizi K, Hosseini M, Nouri Vahid L, Falah M, Hemmati S, Hu H, Tzschach A, Ropers HH, Najmabadi H and Kuss AW: A novel nonsense mutation in TUSC3 is responsible for non-syndromic autosomal recessive mental retardation in a consanguineous Iranian family. Am J Med Genet A 155A: 1976-1980, 2011

8. Garshasbi M, Hadavi V, Habibi H, Kahrizi K, Kariminejad R, Behjati F, Tzschach A, Najmabadi H, Ropers HH and Kuss AW: A defect in the TUSC 3 gene is associated with autosomal recessive mental retardation. Am J Hum Genet 82: 1158-1164, 2008.

9. Bova GS, MacGrogan D, Levy A, Pin SS, Bookstein R and Isaacs WB: Physical mapping of chromosome 8p22 markers and their homozygous deletion in a metastatic prostate cancer. Genomics 35: 46-54, 1996.

10. MacGrogan D, Levy A, Bova GS, Isaacs WB and Bookstein R: Structure and methylation-associated silencing of a gene within a homozygously deleted region of human chromosome band 8p22. Genomics 35: 55-65, 1996.

11. Levy A, Dang UC and Bookstein R: High-density screen of human tumor cell lines for homozygous deletions of loci on chromosome arm 8p. Genes Chromosomes Cancer 24: 42-47, 1999.

12. Mohorko E, Owen RL, Malojčić G, Brozzo MS, Aebi M and Glockshuber R: Structural basis of substrate specificity of human oligosaccharyl transferase subunit N33/Tusc3 and Its role in regulating protein N-glycosylation. Structure 22: 590-601, 2014.

13. Guervós MA, Marcos CA, Hermsen M, Nuño AS, Suárez C and Llorente JL: Deletions of N33, STK11 and TP53 are involved in the development of lymph node metastasis in larynx and pharynx carcinomas. Cell Oncol 29: 327-334, 2007.

14. Pils D, Horak P, Gleiss A, Sax C, Fabjani G, Moebus VJ, Zielinski C, Reinthaller A, Zeillinger R and Krainer M: Five genes from chromosomal band $8 \mathrm{p} 22$ are significantly down-regulated in ovarian carcinoma: N33 and EFA6R have a potential impact on overall survival. Cancer 104: 2417-2429, 2005.

15. Khalid AM, Asano A, Hosaka YZ, Takeuchi T and Yamano Y: Tumor suppressor candidate TUSC3 expression during rat testis maturation. Biosci Biotechnol Biochem 77: 2019-2024, 2013.

16. Horak P, Tomasich E, Vaňhara P, Kratochvílová K, Anees M, Marhold M, Lemberger CE, Gerschpacher M, Horvat R, Sibilia M, et al: TUSC3 loss alters the ER stress response and accelerates prostate cancer growth in vivo. Sci Rep 4: 3739, 2014.

17. National Lung Screening Trial Research Team, Aberle DR, Berg CD, Black WC, Church TR, Fagerstrom RM, Galen B, Gareen IF, Gatsonis C, Goldin J, et al: The national lung screening trial: Overview and study design. Radiology 258: 243-253, 2011.

18. Jemal A, Siegel R, Xu J and Ward E: Cancer statistics, 2010. CA Cancer J Clin 60: 277-300, 2010. 